\title{
Desempenho Produtivo de Vacas de Quatro Grupos Genéticos Submetidas a Diferentes Manejos Alimentares Desmamadas aos 42 ou 63 Dias Pós-Parto
}

\author{
Liliane Cerdótes $^{1}$, João Restle ${ }^{2}$, Ivan Luiz Brondani ${ }^{3}$, Elisa Kohler Osmari ${ }^{4}$, Diogo Carvalho \\ Soccal ${ }^{5}$, Maurício Fernandes dos Santos ${ }^{6}$
}

\begin{abstract}
RESUMO - O objetivo deste trabalho foi avaliar o desempenho produtivo de vacas de corte, das raças Charolês (C), Nelore (N), mestiças CN e NC, mantidas em pastagem nativa, suplementadas com farelo de arroz e desmamadas aos 42 ou 63 dias pós-parto, ou não suplementadas e desmamadas aos 63 dias. A idade das vacas variou de 3 a 12 anos, sendo agrupadas em quatro classes, primíparas, jovens, adultas e velhas. O ganho de peso médio diário (GMD) do parto aos 63 dias pós-parto foi maior para as vacas suplementadas. Vacas desmamadas aos 42 dias apresentaram maior diferença de peso (61,5 kg), do parto ao diagnóstico de gestação, em relação às não suplementadas (45,7 kg), não diferindo das suplementadas desmamadas aos 63 dias (57,8 kg). Do final do período de acasalamento ao diagnóstico de gestação as mestiças CN apresentaram maior GMD (860 g), em relação às C (552 g) e N (648 g), não diferindo das mestiças NC (670 g). Vacas N foram mais leves em relação aos demais grupos genéticos, em todos os períodos. Vacas primíparas e velhas perderam peso, enquanto as jovens e adultas apresentaram leve ganho de peso do parto ao desmame. Vacas suplementadas desmamadas aos 42 dias apresentaram menor intervalo de partos (IP), em relação as desmamadas aos 63 dias (367 contra 384 dias), no entanto, não diferiram quanto à percentagem de parição, respectivamente, 72,0 e 72,2\%. Nas vacas desmamadas aos 63 dias, a suplementação não alterou o IP, mas resultou em aumento significativo na taxa de reprodução (72,2 contra 53,7\%). Vacas adultas e velhas que desmamaram aos 42 dias apresentaram IP inferior a um ano, 356 e 353 dias, respectivamente, apresentaram maiores peso e condição corporal, por ocasião do diagnóstico de gestação, e maior taxa de parição, respectivamente, 80,0 e 76,3\%, contra 41,4 e $57,5 \%$ que o observado nas primíparas e jovens.
\end{abstract}

Palavras-chave: Bos indicus, Bos taurus, cruzamento, desmame precoce, reprodução

\section{Performance of Cows of Four Genetic Groups Submitted to Different Feeding Managements, Weaned at 42 or 63 Days}

\begin{abstract}
The objective of this experiment was to evaluate the performance of Charolais (C), Nellore (N), CN and NC crossbred cows, kept on native pasture, supplemented with rice bran and weaned at 42 or 63 days, or not supplemented and weaned of 63 days. Cow age ranged from 3 to 12 years and was classified into four groups, first calf, young, adult and old cows. Average daily weight gain (ADG) from calving to 63 days postpartum was higher for supplemented cows. Cows weaned at 42 days showed higher weight difference $(61.5 \mathrm{~kg}$ ) from calving to pregnancy diagnostic than the not supplemented cows (45.7 kg), and did not differ from the supplement cows weaned at 63 days $(57.8 \mathrm{~kg})$. From the end of the mating season to the pregnancy diagnostic the CN crossbred cows showed higher ADG (860 g) than the C (552 g) and N (648 g) and did not differ from the NC (670 g). Nellore cows were lighter than the other genetic groups at all periods evaluated. First calf and old cows lost weight, while young and adult cows showed light weight gain from calving to weaning. Supplemented cows weaned at 42 days showed lower calving interval (CI) than those weaned at 63 days (367 vs 384 days), however, did not differ in calving rate, respectively, 72.0 and 72.2\%. For 63 days weaned cows, supplementation did not alter CI, but resulted in significant increase in calving rate (72.2 vs 53.7\%). Adult and old cows weaned at 42 days showed shorter CI than one year, 356 and 353 days, respectively, displayed higher weight and body condition at gestation diagnostic and higher calving rate, respectively, 80.0 and $76.3 \%$, versus 41.4 and $57.5 \%$, observed for first calf and young cows.
\end{abstract}

Key Words: Bos indicus, Bos taurus, crossbreeding, early weaning, reproduction

\footnotetext{
${ }^{1}$ Zootecnista, doutoranda do curso de Pós-Graduação em Zootecnia da FCAV/UNESP. E.mail: cerdotes@fcav.unesp.br

2Engenheiro-Agrônomo, PhD, Pesquisador Visitante/CNPq - Departamento de Produção Animal - UFG. E.mail: jorestle@terra.com.br

3 Zootecnista, Dr., Professor Assistente do Departamento de Zootecnia da UFSM. E.mail: ilbrondani@uol.com.br

${ }^{4}$ Aluna do curso de graduação em Zootecnia da UFSM. E.mail: elisazootecnista@yahoo.com.br

${ }^{5}$ Aluno do curso de graduação em Zootecnia da UFSM. E.mail: diogocsoccal@bol.com.br

${ }^{6}$ Aluno do curso de Agronomia da UFSM. E.mail: maurifs@bol.com.br
} 


\section{Introdução}

A pecuária de corte do Estado do Rio Grande do Sul ainda é considerada uma atividade com baixos índices produtivos, entre eles a taxa de reprodução. Segundo Restle \& Vaz (1998), a taxa de desmame no Rio Grande do Sul é de aproximadamente 55\%. As principais causas deste baixo desempenho são a qualidade e a estacionalidade da pastagem nativa (PN) a que o rebanho de cria é submetido (Costa et al., 1981).

Entre os vários fatores que afetam o desempenho reprodutivo do rebanho de cria, pode-se destacar o meio ambiente, já que reprodução é uma característica de baixa herdabilidade, além do grupo genético e da idade dos ventres.

O baixo desempenho reprodutivo do rebanho bovino é o reflexo do longo intervalo de partos (IP), decorrente do anestro pós-parto prolongado (Dode et al., 1989). O IP é composto pelo tempo de gestação e pelo intervalo do parto à concepção subseqüente. $\mathrm{O}$ tempo de gestação pode variar de 280-290 dias, principalmente de acordo com o grupo genético da vaca e do sexo do bezerro. Como o período de gestação apresenta pouca variação, o principal fator determinante do IP é o intervalo do parto ao primeiro cio pós-parto. Para que uma vaca repita cria dentro dos 365 dias, é necessário que ela conceba no máximo até 85 dias pós-parto. Considerando que, para obter uma taxa de concepção satisfatória, é necessário intervalo de 60 dias do parto ao cio, restam apenas 25 dias para a vaca conceber visando o IP de um ano.

Para aumentar o desempenho do rebanho de cria é imperativo buscar alternativas que visam reduzir o período de anestro pós-parto com a melhoria do meio ambiente.

O nível nutricional pós-parto, assim como a condição corporal (CC) da vaca ao parto e sua evolução, principalmente até o início do período de acasalamento, é de suma importância na retomada da atividade reprodutiva (Short \& Adams, 1988; Short et al., 1990; Williams, 1990). Os efeitos da nutrição atuam via uma complexa interação entre a quantidade e qualidade de nutrientes ingeridos e a competição por nutrientes para outras funções fisiológicas ao lado da reprodução (Short et al., 1990). Segundo Jolly et al. (1995), a subnutrição ou inadequado consumo de nutrientes é o maior fator que contribui para o prolongado anestro pós-parto. Além da subnutrição, a lactação exerce grande influência na retomada da atividade reprodutiva, pois os hormônios da lactação são antagônicos aos hormônios da reprodução, que desencadeiam o ciclo estral.

Segundo Costa et al. (1981), o incremento do nível nutricional das vacas utilizando-se pastagens cultivadas resultou em aumento significativo da taxa de reprodução. No entanto, o seu custo elevado tem limitado a sua utilização.

Outra alternativa para incrementar a taxa de reprodução do rebanho de cria é o desmame antecipado dos bezerros (Restle \& Vaz, 1998). O desmame aos 90 dias ou menos de idade tem resultado no aumento da taxa de reprodução, conforme constatado por vários autores (Moore \& Rocha, 1983; Lobato \& Barcellos, 1992; Moojen et al., 1994; Moletta \& Perotto, 1997; Restle et al., 2001).

O desmame antecipado aos 90 dias beneficia principalmente as vacas que parem na primeira metade da época de parição (Restle \& Vaz, 1998). Visando ampliar o benefício do desmame antecipado, atualmentem tem-se buscado o desmame com menos idade (60-90 dias).

O objetivo do presente estudo foi estudar os efeitos resultantes do desmame aos 63 ou 42 dias de idade associados à suplementação das vacas com farelo de arroz integral no período de aleitamento, ou desmame aos 63 dias sem suplementação, sobre o desempenho produtivo e reprodutivo de vacas de corte mantidas exclusivamente em PN.

\section{Material e Métodos}

O experimento foi conduzido no Setor de Bovinocultura de Corte do Departamento de Zootecnia da Universidade Federal de Santa Maria, Estado do Rio Grande do Sul, no período de setembro de 2001 a dezembro de 2002.

Foi estudado o desempenho produtivo de vacas mantidas em PN, submetidas aos seguintes manejos: Manejo I - Sem suplementação, sendo os bezerros desmamados aos 63 dias de idade; Manejo II Suplementação com farelo de arroz integral, equivalente a $0,7 \%$ do peso vivo das vacas, sendo os bezerros desmamados aos 42 dias de idade; Manejo III - Suplementação com farelo de arroz integral, equivalente a $0,7 \%$ do peso vivo das vacas, sendo os bezerros desmamados aos 63 dias de idade.

Foram utilizados 159 pares de vacas e bezerros, sendo 29 vacas Charolês (C), 22 Nelore (N), 43 mestiças CN filhas de touros C (1/2 C $1 / 2 \mathrm{~N}$ e 3/4 C $1 / 4 \mathrm{~N})$ e 65 
mestiças NC filhas de touros N (1/2 N 1/2 C; 3/4 N 1/4 C e $5 / 8 \mathrm{~N} \mathrm{3/8} \mathrm{C}$ ). As vacas mestiças $\mathrm{CN}$ produziram bezerros filhos de touros $\mathrm{N}$, enquanto as vacas mestiças NC produziram bezerros filhos de touros $\mathrm{C}$.

A idade das vacas variou de 3 a 12 anos, sendo agrupadas em quatro classes, primíparas (3 anos de idade), jovens (4-5 anos de idade), adultas (6-8 anos de idade) e velhas (+ 8 anos de idade). As vacas foram equilibradas nos diferentes manejos, segundo a idade.

Vacas dos manejos II e III foram mantidas juntas até o desmame. Foram utilizados dois potreiros, sendo realizado o rodízio dos animais a cada 14 dias. A lotação média durante o período de aleitamento foi de 0,9 vaca mais bezerro/ha. A massa de forragem disponível foi determinada a cada 28 dias pela técnica de dupla amostragem (Haydock \& Shaw, 1975). Nas amostras de forragem, foram determinados os teores de proteína bruta, matéria mineral e matéria orgânica e as digestibilidades in vitro da matéria seca e da matéria orgânica (AOAC, 1984) e fibra em detergente neutro (FDN), segundo Goering \& Van Soest (1970).

As vacas foram pesadas nas primeiras 24 horas após o parto e, após, com intervalos de 21 dias até os 63 dias pós-parto, existindo variação de \pm 3 dias, de acordo com a data do parto. Após, foram pesadas ao final do período de acasalamento e por ocasião do diagnóstico de gestação, sendo que nos dias das pesagens também foi atribuída a CC, seguindo uma escala de 1-5 (1 = muito magro, 2 = magro, 3 = médio, 4 = gordo e 5 = muito gordo).

Após o desmame, as vacas foram mantidas apenas em PN e colocadas no rodeio de cria para que se realizassem a observação de cios e posterior inseminação artificial. A inseminação ocorreu durante o mês de dezembro de 2001; em seguida, as vacas foram separadas por grupo genético e expostas aos touros, sendo que vacas $\mathrm{N}$ puras e as mestiças filhas de touro $\mathrm{C}$ foram expostas a touro $\mathrm{N}$, ao passo que as vacas $\mathrm{C}$ puras e as mestiças filhas de touros $\mathrm{N}$ foram expostas a touros $C$, até o final do período de acasalamento, no final de fevereiro, momento em que foi obtido o peso do final de monta das vacas e sua CC. No final do mês de abril, 60 dias após o final do período de acasalamento, foi realizado o diagnóstico de gestação, através do toque retal, e nesta ocasião foram tomados o peso e CC.

O IP foi obtido por intermédio da diferença da data do parto em 2001 e a data do parto subseqüente, em 2002.

O delineamento experimental foi inteiramente casualizado em um arranjo fatorial $4 \times 3$ (quatro grupos genéticos e três manejos), com número de repetições variável por tratamento. Os dados coletados foram submetidos à análise de variância, incluindo no modelo estatístico os efeitos de manejo, grupo genético, idade da vaca e as interações entre esses fatores. Ordem de parição foi incluída no modelo como covariável. Para as variáveis em que houve efeito significativo, as médias foram comparadas pelo teste de Tukey, a 5\% de significância. As análises foram realizadas por intermédio do pacote estatístico SAS (1993).

Para a variável dependente peso da vaca ao parto, o efeito de tipo de manejo foi retirado do modelo. A taxa de parição foi analisada pelo qui-quadrado $\left(\chi^{2}\right)$.

\section{Resultados e Discussão}

Na Tabela 1, encontram-se as médias para a massa de forragem e os conteúdos qualitativos da PN de setembro de 2001 a fevereiro de 2002. Observa-se que a disponibilidade da massa de forragem aumentou linearmente de setembro a fevereiro de 2002, de acordo com a taxa de crescimento, que também se elevou linearmente até janeiro, reflexo das melhores condições climáticas para o desenvolvimento da PN. A digestibilidade in vitro da matéria orgânica foi baixa, no início, elevando-se até janeiro e declinando após. A FDN aumentou até janeiro, apresentando leve declínio posteriormente. De maneira geral, os dados qualitativos da PN foram similares aos obtidos por outros autores (Moojen et al., 1994; Alves Filho, 1995).

Na Tabela 2, encontram-se as médias para o GMD das vacas, de acordo com o manejo alimentar no período logo após o parto. Ao comparar vacas desmamadas aos 63 dias, suplementadas ou não, verifica-se que o GMD das vacas não suplementadas foi negativo, sendo de $-94 \mathrm{~g} /$ dia nos primeiros 42 dias, caindo para - 217 g/dia dos 42 aos 63 dias pós-parto. Já nas vacas suplementadas, o GMD, nos mesmos períodos, embora baixo, foi positivo, sendo de 49 e $84 \mathrm{~g} /$ dia. Estes dados corroboram com os obtidos por Moojen et al. (1994), os quais verificaram maior GMD no período de aleitamento em vacas mantidas em pastagem cultivada, quando comparado ao GMD observado nas vacas mantidas em PN.

Ao considerar as duas idades de desmame nas vacas suplementadas, constata-se que aos 21 dias após o desmame estas vacas passaram a ter aumento de peso diário de 283 g, indicando que a supressão da

R. Bras. Zootec., v.33, n.3, p.585-596, 2004 
produção de leite teve reflexo direto no GMD, o que resultou em GMD similar do parto aos 63 dias e do parto ao final do período de acasalamento para ambos os grupos, indicando que a presença do bezerro junto a vaca por mais 21 dias nas vacas desmamadas aos 63 dias, não afeta o GMD nestes intervalos. Estes dados estão de acordo com os dados observados por Moojen et al. (1994), os quais verificaram GMD similar do nascimento aos 213 dias pós-parto para vacas desmamadas aos 101 ou 213 dias. Todavia, como observado no presente estudo, vacas desmamadas aos 101 dias apresentaram maior GMD no período logo após o desmame, em comparação ao GMD das vacas que continuaram com o bezerro ao pé.

O GMD dos 63 dias ao final do período de acasalamento foi similar entre vacas desmamadas aos 63 dias, suplementadas ou não, sendo o GMD de ambos os grupos superior ao das vacas desmamadas aos 42 dias. Esta diferença pode ser explicada em parte pelo ganho de peso compensatório, pois, no período anterior, dos 42 aos 63 dias, as vacas desmamadas aos 42 dias manifestaram GMD significativamente superior ao das não suplementadas e numericamente superior ao das suplementadas com desmame aos 63 dias. Embora o GMD dos 63 dias ao final do período de acasalamento, nas vacas desmamadas aos 63 dias com ou sem suplementação, não tenha apresentado diferença significativa, observa-se que, do parto ao final do período de acasalamento foi superior nas vacas suplementadas, concordando com Costa et al. (1981), os quais, trabalhando com vacas mantidas em pastagem cultivada ou nativa neste período, verificaram maior GMD para aquelas submetidas à pastagem cultivada. Estes autores atribuíram esta diferença no GMD às diferenças qualitativas e quantitativas das pastagens envolvidas.

O ganho de peso do final do acasalamento até o diagnóstico de gestação foi similar entre os três grupos. Verifica-se, ainda na Tabela 2, que o GMD durante todo o período avaliado, do parto ao diagnóstico de gestação, foi superior nas vacas suplementadas e desmamadas aos 42 dias em relação às não suplementadas, sendo que as suplementadas e desmamadas aos 63 dias apresentaram GMD intermediário neste período.

Em relação à CC das vacas, as não suplementadas apresentaram queda na CC (3,01 para 2,94 pontos), acompanhando a queda no GMD. Nas vacas suplementadas e desmamadas aos 63 dias, houve leve acréscimo na CC (3,11 para 3,14 pontos). Nas vacas desmamadas aos 42 dias, a CC manteve-se praticamente igual durante o aleitamento (3,05 para 3,04 pontos), ocorrendo acréscimo no período logo após o desmame.

Tabela 1 - Médias para a massa de forragem disponível (MF), teor de matéria seca (MS), taxa diária de acúmulo de MS (TDA), digestibilidade in vitro da matéria orgânica (DIVMO) e da MS (DIVMS), teores de proteína bruta (PB) e fibra em detergente neutro (FDN) da pastagem nativa

Table 1 - Means for forage mass (FM), dry matter (DM) content, DM daily accumulation rate (DAR), in vitro organic matter (IVODM) and dry matter digestibility (IVDM), crude protein $(C P)$ and neutral detergent fiber(NDF) contents of the native pasture

\begin{tabular}{|c|c|c|c|c|c|c|c|}
\hline \multirow[t]{2}{*}{$\begin{array}{l}\text { Variáveis } \\
\text { Variables }\end{array}$} & \multicolumn{6}{|c|}{$\begin{array}{l}\text { Mês } \\
\text { Month }\end{array}$} & \multirow[t]{2}{*}{$\begin{array}{l}\text { Média } \\
\text { Mean }\end{array}$} \\
\hline & $\begin{array}{l}\text { Setembro } \\
\text { September }\end{array}$ & $\begin{array}{l}\text { Outubro } \\
\text { October }\end{array}$ & $\begin{array}{l}\text { Novembro } \\
\text { November }\end{array}$ & $\begin{array}{l}\text { Dezembro } \\
\text { December }\end{array}$ & $\begin{array}{l}\text { Janeiro } \\
\text { January }\end{array}$ & $\begin{array}{c}\text { Fevereiro } \\
\text { February }\end{array}$ & \\
\hline $\begin{array}{l}\mathrm{MF}, \mathrm{kg} \mathrm{MS} / \mathrm{ha} \\
F M, \mathrm{~kg} D M / \mathrm{ha}\end{array}$ & 2807,57 & 2372,79 & 3506,66 & 4591,95 & 5083,14 & 7078,36 & 4240,08 \\
\hline $\begin{array}{l}\mathrm{MS}, \% \\
D M, \%\end{array}$ & 54,15 & 42,21 & 46,36 & 45,01 & 48,87 & 40,41 & 46,17 \\
\hline $\begin{array}{l}\text { TDA, kg/ha/dia } \\
D A R, k g / h a / d a y\end{array}$ & - & 32,00 & 36,28 & 60,26 & 101,60 & 69,59 & 59,95 \\
\hline $\begin{array}{l}\text { DIVMO, \% } \\
\text { IVODM, \% }\end{array}$ & 14,05 & 27,72 & 35,01 & 33,31 & 36,92 & 31,31 & 29,72 \\
\hline $\begin{array}{l}\text { DIVMS, \% } \\
\text { IVDM, \% }\end{array}$ & 17,21 & 28,45 & 37,06 & 35,18 & 12,06 & 34,25 & 32,37 \\
\hline $\begin{array}{l}\mathrm{PB}, \% \\
C P, \%\end{array}$ & 6,16 & 6,01 & 5,85 & 5,46 & 5,40 & 4,99 & 5,64 \\
\hline $\begin{array}{l}\text { FDN, \% } \\
N D F, \%\end{array}$ & 69,91 & 70,69 & 71,48 & 72,26 & 73,52 & 71,69 & 71,59 \\
\hline
\end{tabular}

R. Bras. Zootec., v.33, n.3, p.585-596, 2004 
No final do período de acasalamento, vacas não suplementadas com desmame aos 63 dias apresentaram CC inferior ao das vacas suplementadas também desmamadas aos 63 dias (3,12 contra 3,31 pontos), concordando com as observações de Stagg et al. (1995), de que vacas mantidas em melhor condição alimentar apresentam melhor CC. As vacas desmamadas aos 42 dias apresentaram CC intermediária (3,19 pontos), não diferindo significativamente das demais.

No diagnóstico de gestação, a CC foi significativamente inferior nas vacas não suplementadas 3,42 pontos contra 3,60 e 3,56 pontos, respectivamente para vacas suplementadas desmamadas aos 63 e aos 42 dias pós-parto. O maior incremento na CC ocorreu do final do acasalamento até o diagnóstico de gestação, reflexo do maior GMD neste período.

Em relação às diferentes idades de desmame, nas vacas suplementadas, não se observou diferença na CC. No entanto, Moojen et al. (1994) e Restle et al.
(2001) verificaram maior CC em vacas desmamadas aos 90 dias, quando comparadas àquelas desmamadas aos 210 dias pós-parto.

Na Tabela 3, são apresentados os pesos médios das vacas, de acordo com o manejo alimentar no período de aleitamento. Não foram verificadas diferenças significativas entre os manejos nos diferentes períodos avaliados. No entanto, Moore \& Rocha (1983), trabalhando com vacas Gir, observaram maior peso ao desmame em vacas desmamadas aos 30 dias, quando comparadas aquelas desmamadas aos 90 dias. Da mesma forma, Pascoal et al. (2000) verificaram decréscimo linear no peso da vaca ao desmame, com o aumento da idade de desmame de bezerras Hereford.

Embora não tenha ocorrido diferença significativa entre os diferentes manejos no peso, por ocasião do diagnóstico de gestação, houve diferença significativa no acréscimo de peso do parto ao diagnóstico de gestação. Vacas desmamadas aos 42 dias tiveram a

Tabela 2 - Médias ajustadas e erros-padrão, em gramas, para o ganho de peso médio diário das vacas do parto aos 42 dias pós-parto (GMDp-42), dos 42 aos 63 dias pós-parto (GMD42-63), do parto aos 63 dias pós-parto (GMDp-63), do parto ao final de monta (GMDpfm), dos 63 dias pós-parto ao final de monta (GMD63-fm) e do final de monta ao diagnóstico de gestação (GMDfmdg), de acordo com o manejo alimentar da vaca

Table 2 - Adjusted means and standard errors, in grams, for cow average daily weight gain, from calving to 42 days post-calving (ADGC-42), from 42 to 63 days post-calving (ADG42-63), from calving to 63 days post-calving (ADGC-63), from calving to the end of mating season (ADGcems), from 63 days to the end of mating season (ADG63ems) and from the end of mating season to gestation diagnostic (ADGemsg), according to cow feeding management

\begin{tabular}{|c|c|c|c|}
\hline \multirow[b]{2}{*}{$\begin{array}{l}\text { Variáveis } \\
\text { Variables }\end{array}$} & \multicolumn{3}{|c|}{$\begin{array}{c}\text { Manejo alimentar das vacas } \\
\text { Cow feeding management }\end{array}$} \\
\hline & $\begin{array}{c}\text { Vacas não } \\
\text { suplementadas } \\
\text { desmamadas aos } \\
63 \text { dias } \\
\text { Non supplemented } \\
\text { cows weaned at } 63 \text { days }\end{array}$ & $\begin{array}{c}\text { Vacas } \\
\text { suplementadas } \\
\text { desmamadas } \\
\text { aos } 63 \text { dias } \\
\text { Supplemented cows } \\
\text { weaned at } 63 \text { days }\end{array}$ & $\begin{array}{c}\text { Vacas } \\
\text { suplementadas } \\
\text { desmamadas } \\
\text { aos } 42 \text { dias } \\
\text { Supplemented cows } \\
\text { weaned at } 42 \text { days }\end{array}$ \\
\hline $\begin{array}{l}\text { GMDp-42 } \\
A D G C-42\end{array}$ & $-94 \pm 54 b$ & $49 \pm 100 \mathrm{a}$ & $-37 \pm 57 a b$ \\
\hline $\begin{array}{l}\text { GMD42-63 } \\
A D G 42-63\end{array}$ & $-217 \pm 91 b$ & $84 \pm 100 a b$ & $283 \pm 96 a$ \\
\hline $\begin{array}{l}\text { GMDp-63 } \\
\text { ADGc-63 }\end{array}$ & $-135 \pm 37 b$ & $60 \pm 40 a$ & $70 \pm 38 a$ \\
\hline $\begin{array}{l}\text { GMDpfm } \\
\text { ADGcems }\end{array}$ & $77 \pm 47 b$ & $194 \pm 30 a$ & $158 \pm 29 a$ \\
\hline $\begin{array}{l}\text { GMD63-fm } \\
\text { ADG63-ms }\end{array}$ & $290 \pm 47 a$ & $331 \pm 48 a$ & $181 \pm 46 b$ \\
\hline $\begin{array}{l}\text { GMDfmdg } \\
\text { ADGemsgd }\end{array}$ & $645 \pm 79 a$ & $604 \pm 73 a$ & 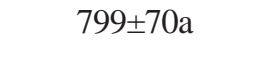 \\
\hline $\begin{array}{l}\text { GMDpdg } \\
\text { ADGcgd }\end{array}$ & $260 \pm 23 b$ & $329 \pm 2 a b$ & $353 \pm 21 a$ \\
\hline
\end{tabular}

Médias na linha, seguidas por letras diferentes, diferem $(P<0,05)$ pelo teste Tukey.

Means, within a row, followed by different letters, differ $(P<.05)$ by Tukey test.

R. Bras. Zootec., v.33, n.3, p.585-596, 2004 
maior diferença $(61,5 \mathrm{~kg})$ em relação às não suplementadas $(45,7 \mathrm{~kg})$, sendo que, nas suplementadas desmamadas aos 63 dias, o acréscimo em peso (57,8 kg) foi intermediário, não diferindo dos animais dos demais manejos. Estas diferenças representaram, respectivamente, 17, 14 e $16 \%$.

O peso no diagnóstico de gestação, bem como a CC, tem implicações de ordem prática importantes. Para vacas que permanecem no rebanho, a melhor CC no outono permite melhor condição para atravessar o período crítico do inverno (Restle et al., 2001). Segundo Restle \& Vaz (1998), quando é realizado o desmame precoce de bezerros, o problema da "vaca fraca" deixa de existir. Vacas com melhor CC na entrada do inverno irão apresentar também melhor CC na primavera subseqüente, durante a parição. Esse melhor estado na parição propicia melhores condições para a produção de leite (Cerdótes et al., 2004) e retorno mais rápido à manifestação de cio (Restle et al., 2001).
Para as vacas de descarte, o desmame precoce tem sido ótimo meio para acelerar a comercialização, pois, parte das vacas que têm CC adequada pode ser comercializadas ou representa menor período de terminação (Pascoal \& Vaz, 1997).

Diferentemente do que foi observado no peso das vacas do trabalho de Moletta \& Perotto (1997), os dados do presente estudo mostraram que o peso das vacas no final de monta não diferiu entre os diferentes manejos pré-desmame. Já Sá et al. (1997), desmamando bezerros com 60 ou 120 dias de idade, verificaram maior peso da vaca ao final do período de acasalamento para aquelas desmamadas aos 60 dias pós-parto. Entretanto, como ocorreu no presente estudo, o GMD neste período foi semelhante para as vacas que tiveram seus bezerros desmamados em diferentes idades.

Na Tabela 4 constam as médias do GMD, em função dos grupos genéticos das vacas. Não houve diferença significativa no GMD do parto ao desmame entre os grupos genéticos estudados, sendo que todos

Tabela 3 - Médias ajustadas e erros-padrão, em kg, para o peso das vacas ao parto (Pparto), aos 42 dias pós-parto (P42), aos 63 dias pós-parto (P63), ao desmame (Pdesm.), ao final de monta (Pfm), no diagnóstico de gestação (Pdg) e ganho de peso do parto ao diagnóstico de gestação (GPpdg), de acordo com o manejo alimentar das vacas

Table 3 - Adjusted means and standard errors, in $\mathrm{kg}$, for cow weight at calving (Wcalv.), at 42 days post-calving (W42), at 63 day post-calving, (W63), at weaning (Wwean.), at the end of mating season (Wems), at gestation diagnostic (Wgd) and gain in weight from calving to gestation diagnostic (GWcgd), according to cow feeding management

\begin{tabular}{|c|c|c|c|}
\hline \multirow[b]{2}{*}{$\begin{array}{l}\text { Variáveis } \\
\text { Variables }\end{array}$} & \multicolumn{3}{|c|}{$\begin{array}{l}\text { Manejo alimentar das vacas } \\
\text { Cow feeding management }\end{array}$} \\
\hline & $\begin{array}{c}\text { Vacas não } \\
\text { suplementadas } \\
\text { desmamadas aos } \\
63 \text { dias } \\
\text { Non supplemented } \\
\text { cows weaned at } 63 \text { days }\end{array}$ & $\begin{array}{c}\text { Vacas } \\
\text { suplementadas } \\
\text { desmamadas } \\
\text { aos } 63 \text { dias } \\
\text { Supplemented cows } \\
\text { weaned at } 63 \text { days }\end{array}$ & $\begin{array}{c}\text { Vacas } \\
\text { suplementadas } \\
\text { desmamadas } \\
\text { aos } 42 \text { dias } \\
\text { Supplemented cows } \\
\text { weaned at } 42 \text { days }\end{array}$ \\
\hline $\begin{array}{l}\text { Pparto } \\
\text { Wcalv. }\end{array}$ & $368,0 \pm 5,9 a$ & $364,4 \pm 6,5 a$ & $375,6 \pm 6,2 a$ \\
\hline $\begin{array}{l}\text { P42 } \\
\text { W42 }\end{array}$ & $364,1 \pm 6,1 \mathrm{a}$ & $366,4 \pm 6,6 a$ & $374,0 \pm 6,3 a$ \\
\hline $\begin{array}{l}\text { P63 } \\
W 63\end{array}$ & $359,5 \pm 6,1 \mathrm{a}$ & $368,2 \pm 6,7 a$ & $380,0 \pm 6,4 a$ \\
\hline $\begin{array}{l}\text { Pdesm. } \\
\text { Wwean. }\end{array}$ & $359,5 \pm 6,1 \mathrm{a}$ & $368,0 \pm 6,7 a$ & $374,0 \pm 6,4 a$ \\
\hline $\begin{array}{l}\text { Pfm } \\
\text { Wems }\end{array}$ & $382,5 \pm 7,1 \mathrm{a}$ & $388,0 \pm 7,1 \mathrm{a}$ & $392,9 \pm 6,8 a$ \\
\hline $\begin{array}{l}\text { Pdg } \\
\text { Wgd }\end{array}$ & $417,7 \pm 8,3 а$ & $422,1 \pm 7,6 a$ & $438,5 \pm 7,4 a$ \\
\hline $\begin{array}{l}\text { GPpdg } \\
\text { GWcgd }\end{array}$ & $45,7 \pm 4,0 \mathrm{~b}$ & $57,8 \pm 3,6 a b$ & $61,5 \pm 3,5 a$ \\
\hline
\end{tabular}

Médias na linha, seguidas por letras diferentes, diferem $(\mathrm{P}<0,05)$ pelo teste Tukey.

Means, within a row, followed by different letters, differ $(P<.05)$ by Tukey test.

R. Bras. Zootec., v.33, n.3, p.585-596, 2004 
Tabela 4 - Médias ajustadas e erros-padrão, em gramas, para o ganho de peso médio diário das vacas do parto ao desmame (GMDpd), do parto ao final de monta (GMDpfm), do desmame ao final de monta (GMDdfm) e do final de monta ao diagnóstico de gestação (GMDfmdg), de acordo com o grupo genético das vacas

Table 4 - Adjusted means and standard errors, in grams, for average daily weight gain (ADG), from calving to weaning ( $A D G C W)$, from calving to the end of mating season (ADGcems), from weaning to the end of mating season (ADGwems) and from the end of mating season to gestation diagnostic (AGDemsgd), according to cow genetic group

\begin{tabular}{|c|c|c|c|c|}
\hline \multirow[t]{2}{*}{$\begin{array}{l}\text { Variáveis } \\
\text { Variables }\end{array}$} & \multicolumn{4}{|c|}{$\begin{array}{l}\text { Grupo genético das vacas } \\
\text { Cow genetic group }\end{array}$} \\
\hline & $\begin{array}{l}\text { Charolês (C) } \\
\text { Charolais (C) }\end{array}$ & $\begin{array}{l}\text { Nelore }(\mathrm{N}) \\
\text { Nellore }(N)\end{array}$ & $\begin{array}{l}\text { Mestiças CN } \\
C N \text { crossbreds }\end{array}$ & $\begin{array}{l}\text { Mestiças NC } \\
\text { NC crossbreds }\end{array}$ \\
\hline $\begin{array}{l}\text { GMDpd } \\
\text { ADGcw }\end{array}$ & $-63 \pm 59 a$ & $-20 \pm 63 a$ & $-62 \pm 48 a$ & $-7 \pm 37 a$ \\
\hline $\begin{array}{l}\text { GMDpfm } \\
\text { ADGcems }\end{array}$ & $125 \pm 37 \mathrm{ab}$ & $127 \pm 43 a b$ & $114 \pm 30 b$ & $207 \pm 23 a$ \\
\hline $\begin{array}{l}\text { GMDdfm } \\
\text { ADGwems }\end{array}$ & $334 \pm 66 a b$ & $215 \pm 76 b$ & $255 \pm 54 b$ & $403 \pm 42 a$ \\
\hline $\begin{array}{l}\text { GMDfmdg } \\
\text { ADGemsgd }\end{array}$ & $552 \pm 106 b$ & $648 \pm 104 b$ & $860 \pm 74 a$ & $670 \pm 58 a b$ \\
\hline
\end{tabular}

apresentaram leve perda de peso neste período. No entanto, Alves Filho (1995) não observou perda de peso em vacas $\mathrm{C}$, $\mathrm{N}$ e de suas respectivas cruzas do parto ao desmame, entretanto, a pastagem era de melhor qualidade.

Embora vacas mestiças tenham produzido mais leite (Cerdótes et al., 2004), não houve diferença em termos de GMD em comparação às puras. A explicação para este comportamento pode estar relacionada ao consumo alimentar, já que Restle et al. (1995) verificaram que animais F1 (1/2 CN 1/2 NC) consumiram mais matéria seca, mesmo quando ajustado para peso metabólico, que animais $\mathrm{C}$ e $\mathrm{N}$ puros. Provavelmente, as vacas mestiças compensaram a maior produção de leite com maior consumo de pasto.

Considerando apenas as vacas mestiças, verifica-se que o GMD do parto ao final do período de acasalamento foi maior nas NC. No entanto, vacas puras apresentaram GMD similar do parto ao final doperíodo de acasalamento, concordando com os dados obtidos por Ribeiro (1989), o qual verificou GMD similar entre vacas puras, Aberdeen Angus e C, do parto ao final do acasalamento. O GMD do final do acasalamento até o diagnóstico de gestação foi superior nas vacas mestiças $\mathrm{CN}$, em relação às puras, não diferindo das mestiças NC.

Houve estreita relação entre o GMD e a CC, concordando com Wiltbank et al. (1964), os quais citam que a CC das vacas varia paralelamente com a variação de peso destas. Em relação às vacas puras, as vacas $\mathrm{C}$ apresentaram CC numericamente inferior do parto ao final do período de acasalamento, em relação às vacas $\mathrm{N}$. Por ocasião do diagnóstico de gestação, esta diferença foi significativa (3,32 contra 3,57 pontos), discordando do que foi constatado por Restle et al. (2001), os quais verificaram, na média, $\mathrm{CC}$ melhor em vacas $\mathrm{C}$ em relação às vacas $\mathrm{N}$. Vacas mestiças CN e NC apresentaram CC similar em todos os períodos, sendo, em média, de 3,2 e 3,3 pontos, citados na mesma ordem.

Em relação ao peso, as vacas $\mathrm{N}$ foram na média as mais leves $(343,8 \mathrm{~kg})$ em todos os períodos avaliados, o que se deve ao seu menor tamanho em relação às vacas $C(393,7 \mathrm{~kg})$ e às mestiças $\mathrm{CN}$ $(411,2 \mathrm{~kg})$ e NC (401,7 kg). Estes resultados corroboram os dados obtidos por Restle et al. (2001), que verificaram, em média, menor peso em vacas $\mathrm{N}$, quando comparadas a vacas C.

Embora não tenha ocorrido diferença significativa entre as vacas mestiças, houve tendência de as vacas CN apresentarem maior peso em todos os períodos, o que, provavelmente, se deve ao fato de serem filhas de touros $\mathrm{C}$, os quais sofreram grande seleção para desenvolvimento muscular. As mestiças NC, por serem filhas de touros N, apresentaram menor peso, devido à ação aditiva dos gens paternos. Alves Filho (1995) constatou maior peso ao parto e ao desmame em vacas $1 / 2 \mathrm{CN}$, quando comparadas às 1/2 NC. Este autor ainda observou heterose de 9,6 e 
$11,8 \%$, respectivamente, para o peso ao parto e ao desmame. Senna (1996) também verificou maior peso das vacas $\mathrm{C}$ em relação às $\mathrm{N}$ e ainda maior peso das mestiças em relação às puras.

$\mathrm{Na}$ Tabela 5, encontram-se as médias para o GMD das vacas, de acordo com a classe de idade. No período do aleitamento, vacas primíparas e velhas perderam peso, vacas adultas praticamente mantiveram o peso e as jovens apresentaram leve ganho. Esta situação reflete a condição fisiológica das vacas, sendo que as primíparas, ainda em fase de crescimento, tiveram que mobilizar reservas corporais para atender à produção de leite e ao crescimento. Já as vacas velhas, que também tiveram que mobilizar reservas corporais durante a lactação, refletiram, em parte, a menor capacidade de coleta de nutrientes (Restle et al., 2001).

Ainda na Tabela 5, verifica-se que a categoria de vacas não afetou o GMD do desmame ao final de monta. No entanto, ao observar maior período, do parto ao final de monta, verifica-se que as diferenças observadas no GMD do parto ao desmame se mantêm, sendo o menor GMD verificado nas vacas primíparas e velhas, seguidas das adultas, as quais apresentaram GMD intermediário. O maior GMD foi observado nas vacas jovens, indicando que, embora o GMD do desmame ao final de monta tenha sido similar entre as diferentes categorias de vacas, a perda de peso das vacas velhas e primíparas do parto ao desmame foi suficiente para que as vacas não conseguissem, no período do parto ao final da monta, ganhos similares aos das vacas jovens e adultas. Estes resultados concordam com os obtidos por Restle et al. (2001), os quais verificaram menor GMD do parto ao final do período de acasalamento nas vacas velhas, ao passo que as vacas jovens e adultas apresentaram GMD similar neste período. No entanto, Costa et al. (1981) não constataram diferença no GMD entre vacas jovens e adultas no intervalo do parto ao início da época de acasalamento. Contudo, estas vacas estavam submetidas a nível nutricional mais elevado que os animais do presente estudo.

A CC das vacas, de acordo com a classe de idade acompanhou sua evolução do peso, concordando com os dados obtidos por Senna (1996), o qual verificou que, quanto mais elevado for o peso, maior é a CC.

Observa-se que vacas primíparas (2,96 pontos) e jovens (3,00 pontos) apresentaram menor CC ao parto em relação às adultas (3,15 pontos) e velhas (3,11 pontos). Houve queda na CC durante o aleitamento nas primíparas e velhas, acompanhando o comportamento do GMD, sendo que ao desmame apresentaram CC de 2,90 e 3,07 pontos, respectivamente. Já nas adultas, não houve variação na CC, ao passo que nas jovens houve leve aumento, apresentando CC ao desmame de 3,03 pontos. Vacas primíparas apresentaram menor CC ao final do acasalamento e por ocasião do diagnóstico de gestação, não havendo diferença entre as demais classes de idade.

Tabela 5 - Médias ajustadas e erros-padrão, em gramas, para o ganho de peso médio diário das vacas do parto ao desmame (GMDpd), do parto ao final de monta (GMDpfm), do desmame ao final de monta (GMDdfm) e do final de monta ao diagnóstico de gestação (GMDfmdg), de acordo com a classe de idade das vacas

Table 5 - Adjusted means and standard errors, in grams, for average daily weight gain, from calving to weaning (ADGCW), from calving to the end of mating season (ADGcems), from weaning to the end of mating season (AGDwems) and from the end of mating season to gestation diagnostic (ADGemsgd), according to cow age class

\begin{tabular}{ccccc}
\hline \multirow{2}{*}{$\begin{array}{c}\text { Variáveis } \\
\text { Variables }\end{array}$} & \multicolumn{4}{c}{$\begin{array}{c}\text { Classe de idade das vacas } \\
\text { Cow age class }\end{array}$} \\
\cline { 2 - 5 } & $\begin{array}{c}\text { Primíparas } \\
\text { First calf }\end{array}$ & $\begin{array}{c}\text { Jovens } \\
\text { Young }\end{array}$ & $\begin{array}{c}\text { Adultas } \\
\text { Adult }\end{array}$ & $\begin{array}{c}\text { Velhas } \\
\text { Old }\end{array}$ \\
\hline GMDpd & $-93 \pm 59 \mathrm{~b}$ & $75 \pm 46 \mathrm{a}$ & $10 \pm 4 \mathrm{ab}$ & $-153 \pm 64 \mathrm{~b}$ \\
ADGcw & $71 \pm 38 \mathrm{~b}$ & $226 \pm 29 \mathrm{a}$ & $180 \pm 26 \mathrm{ab}$ & $95 \pm 44 \mathrm{~b}$ \\
GMDpfm & $243 \pm 67 \mathrm{a}$ & $373 \pm 52 \mathrm{a}$ & $327 \pm 45 \mathrm{a}$ & $265 \pm 78 \mathrm{a}$ \\
$\begin{array}{c}\text { ADGcems } \\
\text { GMDdfm }\end{array}$ & $592 \pm 91 \mathrm{a}$ & $728 \pm 73 \mathrm{a}$ & $704 \pm 64 \mathrm{a}$ & $706 \pm 121 \mathrm{a}$ \\
$\begin{array}{c}\text { ADGwems } \\
\text { GMDfmdg } \\
\text { ADGemsgd }\end{array}$ & & & & \\
\hline
\end{tabular}

Médias na linha, seguidas por letras diferentes, diferem $(P<0,05)$ pelo teste Tukey. Means, within a row, followed by different letters, differ $(P<.05)$ by Tukey test. 
O peso das vacas acompanhou a idade, sendo que as primíparas e as jovens diferiram significativamente entre si e entre as demais classes, não havendo diferença significativa entre adultas e velhas, com pesos médios observados de 321,5; 386,2; 415,0 e $427,7 \mathrm{~kg}$, respectivamente. O maior peso verificado nas vacas velhas e adultas, em parte, pode ser explicado pelo maior tamanho metabólico destes animais, por serem multíparas e também por já terem completado seu crescimento.

Já o menor peso observado nas primíparas devese ao fato de que ainda estão em fase de crescimento. As vacas jovens ainda possuem menor tamanho metabólico, quando comparadas às vacas velhas e adultas, resultando em menor peso. Resultados similares foram observados por Restle et al. (2001), os quais constataram menor peso ao parto, aos 3 e 7 meses e ainda no final do período de acasalamento em vacas de 3-4 anos, quando comparado ao peso de vacas com 57 ou com mais de 8 anos de idade. Alves Filho (1995) também observou menor peso de vacas com 3 anos, seguidas das vacas com 4 e com mais de 4 anos, tanto ao parto como ao desmame.

Houve interação significativa entre o manejo e a classe de idade das vacas para o IP. Observa-se, na Tabela 6, que a suplementação das vacas não afetou o IP, quando acompanhado do desmame aos 63 dias, respectivamente, de 384,7 dias para as vacas não suplementadas e de 384,2 dias para as suplementadas. Estes dados discordam dos resultados obtidos por Moore \& Rocha (1983), Richards et al. (1986) e Lobato \& Barcellos (1992), os quais observaram que vacas mantidas em melhor condição nutricional apresentam menor intervalo do parto ao primeiro cio pósparto, principalmente quando associado à melhor CC.

Em relação às diferentes idades de desmame, observa-se que, na média, as vacas desmamadas aos 63 dias apresentaram maior IP, enquanto a redução da idade ao desmame em 21 dias proporcionou declínio de 17 dias no IP. O desmame aos 42 dias permitiu que estas vacas produzissem um bezerro ao ano, indicando que, nesta fase, a presença do bezerro ao pé da vaca está intimamente associada à retomada de sua atividade cíclica, concordando com Short et al. (1972) e Yavas \& Walton (2000), os quais citam que a presença do bezerro junto à mãe aumenta significativamente o intervalo de anestro pós-parto, que, por conseqüência, aumenta o IP.

O menor IP foi constatado nas vacas suplementadas, velhas e adultas, desmamadas aos 42 dias, sendo os únicos grupos de vacas que conseguiram apresentar IP dentro de um ano, além de serem animais que apresentaram maior peso durante o período experimental.

O maior IP observado nas vacas primíparas suplementadas e desmamadas aos 63 dias pós-parto não era esperado, pois, como estes animais permane-

Tabela 6 - Médias ajustadas e erros-padrão, em dias, para o intervalo de partos das vacas de acordo com o manejo alimentar e a classe de idade das vacas

Table 6 - Adjusted means and standard errors, in days, for cows calving interval, according to cow feeding management and age class

\begin{tabular}{|c|c|c|c|c|}
\hline \multirow[b]{2}{*}{$\begin{array}{l}\text { Classe de idade } \\
\text { das vacas } \\
\text { Cow age } \\
\text { class }\end{array}$} & \multicolumn{4}{|c|}{$\begin{array}{l}\text { Manejo alimentar das vacas } \\
\text { Cow feeding management }\end{array}$} \\
\hline & $\begin{array}{c}\text { Vacas não } \\
\text { suplementadas } \\
\text { desmamadas aos } \\
63 \text { dias } \\
\text { Non supplemented } \\
\text { cows weaned at } 63 \text { days }\end{array}$ & $\begin{array}{c}\text { Vacas } \\
\text { suplementadas } \\
\text { desmamadas } \\
\text { aos } 63 \text { dias } \\
\text { Supplemented cows } \\
\text { weaned at } 63 \text { days }\end{array}$ & $\begin{array}{c}\text { Vacas } \\
\text { suplementadas } \\
\text { desmamadas } \\
\text { aos } 42 \text { dias } \\
\text { Supplemented cows } \\
\text { weaned at } 42 \text { days }\end{array}$ & $\begin{array}{l}\text { Média } \\
\text { Mean }\end{array}$ \\
\hline $\begin{array}{l}\text { Primíparas } \\
\text { First calf }\end{array}$ & $379,6 \pm 18,9 a b$ & $402,3 \pm 9,9 a$ & $377,7 \pm 5,8 a b c$ & $386,5 \pm 8,2$ \\
\hline $\begin{array}{l}\text { Jovens } \\
\text { Young }\end{array}$ & $387,2 \pm 6,3 a b$ & $379,1 \pm 4,2 \mathrm{bc}$ & $382,9 \pm 8,0 \mathrm{bc}$ & $383,0 \pm 3,7$ \\
\hline $\begin{array}{l}\text { Adultas } \\
\text { Adult }\end{array}$ & $376,8 \pm 5,0 \mathrm{bc}$ & $381,1 \pm 4,1$ bc & $356,3 \pm 4,1 \mathrm{c}$ & $371,4 \pm 2,6$ \\
\hline $\begin{array}{l}\text { Velhas } \\
\text { Old }\end{array}$ & $395,3 \pm 8,6 a b c$ & $374,4 \pm 5,3$ bc & $353,0 \pm 7,2 c$ & $374,3 \pm 5,0$ \\
\hline $\begin{array}{l}\text { Média } \\
\text { Mean }\end{array}$ & $384,7 \pm 5,4$ & $384,2 \pm 3,2$ & $367,5 \pm 3,2$ & \\
\hline
\end{tabular}

Médias na linha e na coluna, seguidas por letras diferentes, diferem $(P<0,05)$ pelo teste Tukey.

Means, within a row and collumn, followed by different letters, differ $(P<.05)$ by Tukey test.

R. Bras. Zootec., v.33, n.3, p.585-596, 2004 
ceram durante o período de aleitamento em melhores condições alimentares, esperava-se que apresentassem IP igual ao das vacas desta categoria que não receberam suplementação.

O grupo genético da vaca não afetou o IP, sendo que, em média, foi de 378,8 dias, podendo-se inferir que o período parto concepção dos animais do presente estudo foi, em média, de 94 dias.

Na Tabela 7 constam as médias referentes à taxa de parição no período subsequente, de acordo com o manejo e a classe de idade das vacas. Era esperado melhor desempenho reprodutivo das vacas em todos os manejos alimentares, tendo em vista a remoção precoce dos bezerros. A provável explicação para o baixo desempenho reprodutivo pode ter sido em função do excessivo manejo ao qual os animais foram expostos, tendo em vista que as vacas foram submetidas à ordenha em intervalos de 21 dias e, após os primeiros 21 dias do primeiro parto, uma vez por semana era realizada a separação dos pares de vacas e bezerros que deveriam ser pesados e ordenhados.

Verifica-se que a percentagem de parição acompanhou o incremento da idade da vaca, sendo que nas vacas velhas a taxa de parição foi $93 \%$ superior ao das primíparas. Constata-se também que a suplementação, independentemente da idade de desmame, aumentou significativamente a taxa de parição, o que foi resultado do maior GMD e CC verificados nestes animais, até os 63 dias pós-parto, concordando com os dados obtidos por Costa et al. (1981), Rutter \& Randel (1984), Osoro \& Wright (1992), Moojen et al. (1994), Marques et al. (2001), os quais observaram melhor desempenho reprodutivo nas vacas que ganharam peso mais rapidamente após o parto.

Costa et al. (1981) consideram mais importante a intensidade de ganho de peso do parto ao início do período de monta subseqüente do que o ganho de peso durante a época de monta. Wiltbank et al. (1958) verificaram que, quanto mais alto o ganho de peso após o parto, melhor será o desempenho reprodutivo das vacas. Já Hougton et al. (1990) verificaram maior taxa de prenhez nas vacas com maior CC. Esses autores, comparando o desempenho reprodutivo de vacas desmamadas aos 30 dias ou aos 7 meses pós-parto, submetidas a baixo ou médio nível de energia na dieta no período pré-parto, verificaram taxa de prenhez de 62,5 e $88,9 \%$ para vacas submetidas ao desmame precoce e de 26,7 e 13,3\% para aquelas desmamadas aos 7 meses pósparto, submetidas, respectivamente, a baixo e médio nível de energia no pré-parto. A maior eficiência reprodutiva das vacas suplementadas deve-se em parte à maior CC apresentada por estes animais ao desmame, concordando com Fonseca (1984).

Tabela 7 - Médias para a taxa de parição (\%), de acordo com o manejo alimentar e a classe de idade das vacas

Table 7 - Means for calving rate (\%), according to cow feeding management and age class

\begin{tabular}{|c|c|c|c|c|}
\hline \multirow[b]{2}{*}{$\begin{array}{c}\text { Classe de idade } \\
\text { das vacas } \\
\text { Cow age } \\
\text { class }\end{array}$} & \multicolumn{4}{|c|}{$\begin{array}{c}\text { Manejo alimentar das vacas } \\
\text { Cow feeding management }\end{array}$} \\
\hline & $\begin{array}{c}\text { Vacas não } \\
\text { suplementadas } \\
\text { desmamadas aos } \\
63 \text { dias } \\
\text { Non supplemented } \\
\text { cows weaned at } 63 \text { days }\end{array}$ & $\begin{array}{c}\text { Vacas } \\
\text { suplementadas } \\
\text { desmamadas } \\
\text { aos } 63 \text { dias } \\
\text { Supplemented cows } \\
\text { weaned at } 63 \text { days }\end{array}$ & $\begin{array}{c}\text { Vacas } \\
\text { suplementadas } \\
\text { desmamadas } \\
\text { aos } 42 \text { dias } \\
\text { Supplemented cows } \\
\text { weaned at } 42 \text { days }\end{array}$ & $\begin{array}{l}\text { Média } \\
\text { Mean }\end{array}$ \\
\hline $\begin{array}{l}\text { Primíparas } \\
\text { First calf }\end{array}$ & 9,1 & 50,0 & 70,0 & $41,4 \mathrm{~B} * *$ \\
\hline $\begin{array}{l}\text { Jovens } \\
\text { Young }\end{array}$ & 50,0 & 73,3 & 45,5 & $57,5 \mathrm{~B}$ \\
\hline $\begin{array}{l}\text { Adultas } \\
\text { Adult }\end{array}$ & 70,0 & 73,7 & 85,0 & $76,3 \mathrm{~A}$ \\
\hline $\begin{array}{c}\text { Velhas } \\
\text { Old }\end{array}$ & 77,8 & 83,3 & 77,8 & $80,0 \mathrm{~A}$ \\
\hline $\begin{array}{l}\text { Média } \\
\text { Mean }\end{array}$ & $53,7 b *$ & $72,2 \mathrm{a}$ & $72,0 \mathrm{a}$ & \\
\hline
\end{tabular}

* Médias na linha, seguidas por letras minúsculas diferentes, diferem $(P<0,05)$ pelo teste qui-quadrado $\left(\chi^{2}\right)$.

* Means, within a row, followed by small different letters, differ $(P<.05)$ by Chi-square $\left(\chi^{2}\right)$ test.

** Médias na coluna, seguidas por letras maiúsculas diferentes, diferem $(P<0,05)$ pelo teste qui-quadrado $\left(\chi^{2}\right)$.

** Means, within a collum, followed by capital different letters, differ $(P<.05)$ by Chi-square $\left(\chi^{2}\right)$ test. 
Observa-se ainda que a redução da idade de desmame de 63 para 42 dias, embora tenha tido efeito positivo sobre o IP, não afetou a taxa de parição das vacas. Restle et al. (2001) verificaram incremento de $80 \%$ na taxa de prenhez de vacas desmamadas aos 3 meses de idade, quando comparadas àquelas desmamadas aos 7 meses. Os dados do presente estudo indicam que a partir de certa redução da idade do bezerro ao desmame, a abrupta interrupção da lactação já não possui efeito sobre a taxa de parição subseqüente de vacas suplementadas do parto ao desmame.

O suplemento teve maior efeito sobre a taxa de parição principalmente nas vacas primíparas, em relação aos demais grupos de vacas, pois ocorreu incremento de 40,9 pontos percentuais na taxa de parição das vacas desmamadas aos 63 dias recebendo suplementação, quando comparadas às não suplementadas, sendo as taxas de prenhez, respectivamente, de 50,0 e 9,1\%. Observa-se que, nas primíparas, o desmame aos 42 dias proporcionou incremento de $40,0 \%$ na taxa de parição, quando comparada a de vacas suplementadas desmamadas aos 63 dias pós-parto, evidenciando que a suplementação e a redução na idade ao desmame têm grande importância para o aumento da taxa de parição das vacas primíparas, mesmo quando se trata de idades ao desmame bem precoces e a diferença entre os animais seja de apenas 21 dias. Este fato concorda com Restle et al. (2001), os quais verificaram maior incremento na taxa de prenhez em vacas primíparas, a qual passou de 12,5 para $63,6 \%$, quando a idade de desmame foi reduzida de sete para três meses, em relação aos demais grupos de vacas. Moletta \& Perotto (1997) verificaram que o efeito do desmame precoce foi bastante acentuado na categoria de primíparas, sendo a taxa de prenhez de 82,35 e 3,70\% para as desmamadas aos 70 ou 210 dias, evidenciando que, quanto maior a diferença de idade entre os desmames, mais acentuados são os benefícios a favor do desmame antecipado.

Não houve efeito significativo do grupo genético da vaca, nem interação significativa entre este e o manejo alimentar para taxa de parição. A média dos diferentes grupos genéticos foi de 51\%, ou seja, muito abaixo do esperado, provavelmente devido ao manejo excessivo; resultados semelhantes ao verificado por Moojen et al. (1994), os quais constataram taxa de prenhez média de 67,2 e $37,3 \%$, respectivamente, para vacas desmamadas aos 3 e 7 meses.

R. Bras. Zootec., v.33, n.3, p.585-596, 2004

\section{Conclusões}

A suplementação beneficiou o ganho de peso das vacas do parto aos 63 dias pós-parto.

A taxa de parição, que foi incrementada com a suplementação das vacas, não foi afetada pela idade de desmame dos bezerros.

A suplementação associada ao desmame aos 42 dias, resultou em menor intervalo de partos.

Vacas primíparas apresentaram menor ganho de peso, peso vivo e pior condição corporal, resultando em menor taxa de parição.

As vacas que foram mais beneficiadas, na taxa de parição, pela suplementação e redução da idade de desmame dos bezerros foram as primíparas.

\section{Literatura Citada}

ALVES FILHO, D.C. Evolução do peso e desempenho anual de um rebanho de cria constituído por fêmeas de diferentes grupos genéticos. Santa Maria: Universidade Federal de Santa Maria, 1995. 183p. Dissertação (Mestrado em Zootecnia) - Universidade Federal de Santa Maria, 1995.

ASSOCIATION OF OFFICIAL ANALYTICAL CHEMISTS AOAC. Official methods of analysis. 14.ed. Washington, D.C.: 1984. 1141p.

CERDÓTES, L.; RESTLE, J.; ALVES FILHO, D.C. et al. Produção e composição do leite de vacas de quatro grupos genéticos submetidas a dois manejos alimentares no período de lactação. Revista Brasileira de Zootecnia, v.33, n.3, p.610-622, 2004.

COSTA, A.M.; RESTLE, J.; MULLER, L. Influência da pastagem cultivada no desempenho reprodutivo de vacas com cria ao pé. Revista do Centro de Ciências Rurais, v.11, n.4, p.187-200, 1981.

DODE, M.A.N.; VALLE, E.R.; ROSA, G.O. Efeito da interrupção temporária do aleitamento sobre a fertilidade de vacas de corte. Revista Brasileira de Reprodução Animal, v.13, n.2, p.109-119, 1989.

FONSECA, V.O. O manejo da reprodução e o aumento da eficiência reprodutiva do zebu. Informe Agropecuário, v.10, n.112, p.56-68, 1984.

GOERING, H.K.; Van SOEST, P.J. Forage fiber analysis: apparatus reagents, procedures and some applications. Washington, D. C.: 1970. (Agricultural Handbook, 379)

HAYDOCK, R.M.; SHAW, N.H. The comparative yield method for estimating dry matter yield of pasture. Australian Journal Experimental Agriculture Animal Husbandry, v.15, p.663-669, 1975.

HOUGHTON, P.L.; LEMENAGER, R.P.; HORSTMAN, L.A. et al. Effects of body composition, pre and postpartum energy level and early weaning on reproductive performance of beef cows and preweaning calf gain. Journal of Animal Science, v.68, p.14381446, 1990.

JOLLY, P.D.; McDOUGALL, S.; FITZPATRICK, L.A. et al. Physiological effects of undernutrition on postpartum anoestrus in cows. Journal of Reproduction and Fertility, Supplement 49, p.477-492, 1995. 
LOBATO, J.F.P.; BARCELLOS, J.O.J. Efeito da utilização de pastagem melhorada no pós-parto e do desmame aos 100 ou 180 dias de idade no desempenho reprodutivo de vacas de corte. Revista da Sociedade Brasileira de Zootecnia, v.21, n.3, p.385-395, 1992.

MARQUES, L.P.A.; LOBATO, J.F.P.; SCHENKEL, F.S. Efeito da idade de desmame no desempenho reprodutivo de vacas de corte. In: REUNIÃO ANUAL DA SOCIEDADE BRASILEIRA DE ZOOTECNIA, 38., 2001, Piracicaba. Anais ... Piracicaba: Sociedade Brasileira de Zootecnia, 2001. p.414-415.

MOLLETA, J.L.; PEROTTO, D. Efeito do desmame aos 70 e 210 dias sobre o desempenho ponderal e reprodutivo de vacas de corte. In: REUNIÃO ANUAL DA SOCIEDADE BRASILEIRA DE ZOOTECNIA, 34., 1997, Juiz de Fora. Anais... Juiz de Fora: Sociedade Brasileira de Zootecnia, 1997. p.397-399.

MOOJEN, J.G.; RESTLE, J.; MOOJEN, E.L. Efeito da época da desmama e da pastagem no desempenho de vacas e terneiros de corte. 1- Desempenho das vacas. Revista do Centro de Ciências Rurais, v.24, n.2, p.393-397, 1994.

MOORE, C.P.; ROCHA, C.M.C. Reproductive performance of Gyr cows: the effect of weaning age of calves and postpartum energy intake. Journal of Animal Science, v.57, n.4, p.807-814, 1983.

OSORO, K.; WRIGHT, I.A. The effect of body condition, live weight, breed, age, calf performance, and calving date on reproductive performance of spring-calving beef cows. Journal of Animal Science, v.70, p.1661-1666, 1992.

PASCOAL, L.L.; RESTLE, J.; VAZ, F.N. et al. Efeito da idade e peso das bezerras e produção de leite da vaca sobre o desempenho de bezerras de corte desmamadas precocemente. In: REUNIÃO ANUAL DA SOCIEDADE BRASILEIRA DE ZOOTECNIA, 37., 2000, Viçosa, MG. Anais ... Viçosa, MG: Sociedade Brasileira de Zootecnia, 2000. p.163.

PASCOAL, L.L.; VAZ, F.N. Desmame precoce aos sessenta dias. In: RESTLE, J.; BRONDANI, I.L.; PASCOAL, L.L. et al. (Eds.) Técnicas avançadas na recria e engorda em bovinos de corte. Santa Maria: Universidade Federal de Santa Maria, 1997. p.22-34.

RESTLE, J.; FELTEN, H.G.; VAZ, F.N. Efeito da raça e heterose para desempenho em confinamento de novilhos de corte. Revista Argentina de Produção Animal, v.15, p.852-854, 1995.

RESTLE, J.; VAZ, F.N. Desmame precoce de terneiros. In: SIMPÓSIO INTERNACIONAL SOBRE PRODUÇÃO INTENSIVA DE GADO DE CORTE, 2., 1998, São Paulo. Anais... São Paulo: 1998. p.3-9.

RESTLE, J.; VAZ, R.Z.; ALVES FILHO, S.C. Desempenho de vacas Charolês e Nelore desterneiradas aos três ou sete meses. Revista Brasileira de Zootecnia, v.30, n.2, p.499-507, 2001.

RIBEIRO, E.L.A. Influência de diferentes seqüências de pastagens na produção de leite e no desempenho de vacas de dois grupos genéticos. Santa Maria: Universidade Federal de Santa Maria, 1989. 149p. Dissertação (Mestrado em Zootecnia) - Universidade Federal de Santa Maria, 1989.
RICHARDS, M.W.; SPITZER, J.C.; WARNER, M.B. Effect of varying level of postpartum nutrition and body condition at calving on subsequent reproductive performance in beef cattle. Journal of Animal Science, v.62, p.300-306, 1986.

RUTTER, L.M.; RANDEL, R.D. Postpartum nutrient intake and body condition: effect on pituitary functionand onset of estrus in beef cattle. Journal of Animal Science, v.58, n.2, p.265-274, 1984.

SÁ, I.G.; BARCELLOS, J.O.J.; LOBATO, F.F.P. et al. Efeito do desmame precoce sobre a variação de peso de vacas e bezerros de corte em três sub-épocas de parição. In: REUNIÃO ANUAL DA SOCIEDADE BRASILEIRA DE ZOOTECNIA, 34., 1997, Juiz de Fora. Anais... Juiz de Fora: Sociedade Brasileira de Zootecnia, 1997. p.400-402.

STATISTICAL ANALISYS SYSTEMS - SAS. User's guide. Version 6, Cary: 1993. 1042p.

SENNA, D.B. Desempenho reprodutivo e produção de leite de vacas de quatro grupos genéticos, desterneiradas precocemente, submetidas a diferentes períodos de pastagem cultivada. Santa Maria: Universidade Federal de Santa Maria, 1996. 85p. Dissertação (Mestrado em Zootecnia) - Universidade Federal de Santa Maria, 1996.

SHORT, R.E.; ADAMS, D.C. Nutritional and hormonal interrelationships in beef cattle reproduction. Canadian Journal of Animal Science, v.68, p.29-39, 1988.

SHORT, R.E.; BELLOWS, R.S.; MODDY, E.L. et al. Effects of suckling and mastectomy on bovine postpartum reproduction. Journal of Animal Science, v.74, n.2, p.70, 1972.

SHORT, R.E.; BELLOWS, R.A.; STAIGMILLER, R.B. et al. Physiological mechanisms controlling anestrous and infertility in postpartum beef cattle. Journal of Animal Science, v.68, n.2, p.799-816, 1990.

STAGG, K.; DISKIN, M.G.; SREENAN, J.M. et al. Follicular development in long-term anoestous suckler beef cows fed two levels of energy postpartum. Animal Reproduction Science, v.38, p.49-61, 1995.

WILLIAMS, G.L. Suckling as a regulator of postpartum rebreeding in cattle: a review. Journal of Animal Science, v.68, p.831-852, 1990.

WILTBANK, J.N.; ROWDEN, W.W.; INGALLS, J.E. et al. Influence of post parthum energy level on reproductive performance of Hereford cows restricted in energy intake prior to calving. Journal of Animal Science, v.17, n.4, p.1211, 1958.

WILTBANK, J.N.; ROWDEN, W.W.; INGALLS, J.E. et al. Influence of post-partum energy level on reproductive performance of Hereford cows restricted in energy intake prior to calving. Journal of Animal Science, v.23, p.1049-1053, 1964.

YAVAS, Y.; WALTON, J.S. Postpartum acyclicity in suckled beef cows: a review. Theriogenology, v.54, p.25-55, 2000.

Recebido em: 12/03/03 Aceito em: 01/08/03 